PROCEEDINGS OF THE

AMERICAN MATHEMATICAL SOCIETY

Volume 129, Number 9, Pages 2517-2527

S 0002-9939(01)06176-7

Article electronically published on April 17, 2001

\title{
CLASSIFICATION OF TORSION-FREE GENUS ZERO CONGRUENCE GROUPS
}

\author{
ABDELLAH SEBBAR
}

(Communicated by Dennis A. Hejhal)

\begin{abstract}
We study and classify all torsion-free genus zero congruence subgroups of the modular group.
\end{abstract}

\section{INTRODUCTION}

In this paper we give the explicit list of all the genus 0 congruence subgroups of the modular group with no elliptic elements. This study was motivated by previous work in collaboration with John McKay, 2] 3], in which we studied the properties of Hauptmoduls originating from Moonshine under the action of the Schwarzian derivative.

Given a genus 0 Fuchsian group of the first kind acting on the upper-half plane $\mathfrak{H}$, and a Hauptmodul $f$ for $G$, the Schwarzian derivative $\{f, \tau\}, \tau \in \mathfrak{H}$, is a weight 4 automorphic form. For example, if $G=\Gamma(2)$ and $\lambda$ is the Klein elliptic modular function (which is a Hauptmodul for $\Gamma(2)$ ), then we can show easily that

$$
\frac{1}{\pi^{2}}\{f, \tau\}=E_{4}(\tau)
$$

where $E_{4}$ is the Eisenstein series of weight 4 . Now $E_{4}$ is a modular form for the full modular group and it is holomorphic everywhere, including at the cusps. In fact, for a general group $G$ and a Hauptmodul $f$, one can show that $\{f, \tau\}$ is a weight 4 automorphic form for the normalizer of $G$ in $\mathrm{PSL}_{2}(\mathbb{R})$, with a pole of order 2 at each elliptic fixed point and which is holomorphic everywhere else including at the cusps. Thus, the fact that $\{f, \tau\}$ is holomorphic everywhere is equivalent to $G$ being torsion-free. Investigating these facts for Hauptmoduls originating from Moonshine, for instance those corresponding to $\Gamma_{0}(n)$ for $n=4,6,8,9,12,16,18$ (these are the only ones of this form with genus 0 and no elliptic elements), it turns out that the Schwarzian derivative of the Hauptmoduls of these groups coincides with theta functions of some easily described rank 8 lattices (notice that $E_{4}$ is the theta function of the root lattice $\mathbf{E}_{8}$ ). This phenomenon is still true for the principal congruence groups $\Gamma(n)$ which are genus 0 and torsion-free (namely for $n=2,3,4,5$ ); each has a Schwarzian derivative equal to $\pi^{2} E_{4}$ because their normalizer is the full modular group and because the space of weight 4 modular forms for the full modular group is 1-dimensional. More interestingly, this is still

Received by the editors November 20, 1999.

2000 Mathematics Subject Classification. Primary $20 \mathrm{H} 05$.

Key words and phrases. Automorphic forms, congruence subgroups, genus 0, torsion-free.

(C)2001 American Mathematical Society 
true for those groups of the form $\Gamma_{1}(n)$, namely for $n=4,5,6,7,8,9,10,12$ (these are the only ones). To better understand this phenomenon, we were interested in determining all the congruence subgroups of the modular group which are torsionfree and genus 0 as explicitly as possible (if we drop the congruence condition, then there are infinitely many conjugacy classes of such subgroups).

From another point view, it is shown in [5] that a congruence subgroup of $\mathrm{PSL}_{2}(\mathbb{R})$ which is genus 0 and torsion-free is necessarily conjugate to a congruence subgroup of $\mathrm{PSL}_{2}(\mathbb{Z})$. Thus, the classification in $\mathrm{PSL}_{2}(\mathbb{Z})$ yields the classification in $\mathrm{PSL}_{2}(\mathbb{R})$ and as a consequence gives a positive answer to some questions raised in [2] 3] related to the classification of the Moonshine groups which are torsion-free and genus 0 .

The purpose of this paper is to carry out the classification inside $\mathrm{PSL}_{2}(\mathbb{Z})$. This is done by studying the cusp widths. The main idea is that if a genus 0 torsionfree subgroup of the modular group shares the same cusp widths with one of its subgroups, then it must be equal to it, as such a group can be generated by a set of parabolic elements only. Hence if we start with a congruence group of level $m$, this descent will take us close to $\Gamma(m)$; the result is a set of easily described groups which were introduced by $\mathrm{H}$. Larcher in [1]. Filtering those groups which are torsion-free and genus 0 , we obtain 33 conjugacy classes inside $\mathrm{PSL}_{2}(\mathbb{Z})$ which are partitioned into $15 \mathrm{PSL}_{2}(\mathbb{R})$-conjugacy classes.

\section{Congruence groups}

The content of this section is basic and can be found for instance in [6] and [4]. Let $\mathrm{PSL}_{2}(\mathbb{Z})$ be the modular group which consists of the transformations

$$
z \rightarrow \frac{a z+b}{c z+d} \quad \text { with } a, b, c, d \in \mathbb{Z}, a d-b c=1 .
$$

For convenience, the modular group and its subgroups will be represented by matrices with the understanding that a matrix and its negative will be identified. The principal congruence subgroup of level $m, m$ being a positive integer, is defined by

$$
\Gamma(m)=\left\{A \in \mathrm{PSL}_{2}(\mathbb{Z}), \quad A \equiv \pm I \quad \bmod m\right\} /\{ \pm I\} .
$$

A congruence subgroup of level $m$ of the modular group is a subgroup which contains $\Gamma(m)$ for some positive integer $m$ and does not contain any $\Gamma(n)$ for $n<m$. Examples of such groups are

$$
\begin{gathered}
\Gamma_{1}(m)=\left\{A \in \mathrm{PSL}_{2}(\mathbb{Z}), \quad A \equiv \pm\left(\begin{array}{ll}
1 & * \\
0 & 1
\end{array}\right) \quad \bmod m\right\} /\{ \pm I\}, \\
\Gamma_{0}(m)=\left\{\left(\begin{array}{ll}
a & b \\
c & d
\end{array}\right) \in \mathrm{PSL}_{2}(\mathbb{Z}), \quad c \equiv 0 \bmod m\right\} /\{ \pm I\} .
\end{gathered}
$$

The indices of these groups in the modular group are given by

$$
\left[\mathrm{PSL}_{2}(\mathbb{Z}): \Gamma(2)\right]=6, \quad\left[\mathrm{PSL}_{2}(\mathbb{Z}): \Gamma_{1}(2)\right]=3 \text { and }
$$




$$
\begin{aligned}
& \mu(m):=\left[\mathrm{PSL}_{2}(\mathbb{Z}): \Gamma(m)\right]=\frac{m^{3}}{2} \prod_{\substack{p \mid m \\
p \text { prime }}}\left(1-\frac{1}{p^{2}}\right), m \geq 3, \\
& \mu_{1}(m):=\left[\mathrm{PSL}_{2}(\mathbb{Z}): \Gamma_{1}(m)\right]=\frac{m^{2}}{2} \prod_{\substack{p \mid m \\
p \text { prime }}}\left(1-\frac{1}{p^{2}}\right), m \geq 3, \\
& \mu_{0}(m):=\left[\mathrm{PSL}_{2}(\mathbb{Z}): \Gamma_{0}(m)\right]=m \prod_{\substack{p \mid m \\
p \text { prime }}}\left(1+\frac{1}{p}\right), m \geq 1 .
\end{aligned}
$$

Each congruence group $\Gamma$ acts on the upper-half plane $\mathfrak{H}=\{\tau \in \mathbb{C}, \operatorname{Im}(\tau)>0\}$ in the usual way. If a nonidentity transformation in $\Gamma$ corresponds to a matrix $A$, then $A$ has a fixed point in $\mathfrak{H}$ if $|\operatorname{tr}(A)|$ is 0 (resp. 1); in this case $A$ is of order 2 (resp. 3). Such a transformation is called elliptic, and so is the fixed point. If $|\operatorname{tr}(A)|=2$, then $A$ has a single fixed point on the real line, and $A$ has infinite order; the transformation is called parabolic and the fixed point is called a cusp. While if $|\operatorname{tr}(A)|>2$, the transformation is called hyperbolic, and there are two fixed points on the real line.

The set of parabolic fixed points for $\Gamma$ is $\mathbb{Q} \cup\{\infty\}$. The quotient space $\Gamma \backslash \mathfrak{H}$ can be made into a compact Riemann surface by adjoining the cusps. The genus of this Riemann surface is also called the genus of the group $\Gamma$. The genus of a subgroup of $\Gamma$ is at least the genus of $\Gamma$. The genus of $\Gamma(m)$ is zero if and only if $1 \leq m \leq 5$. The genus of $\Gamma_{1}(m)$ is zero if and only if $m=1, \ldots, 10$ or 12 . The genus of $\Gamma_{0}(m)$ is zero if and only if $m=1, \ldots, 10,12,13,16,18$ or 25 .

Finally, if $r$ is a cusp for $\Gamma$, the stabilizer of $r$ in $\Gamma$ is a subgroup of finite index in the stabilizer of $r$ in $\mathrm{PSL}_{2}(\mathbb{Z})$, and both are infinite cyclic. The index between the stabilizers is called the width of the cusp $r$ in $\Gamma$. Conjugate cusps in $\Gamma$ have the same width and the sum of widths of all the inequivalent classes of cusps is equal to the index of $\Gamma$ in $\operatorname{PSL}_{2}(\mathbb{Z})$; see Chapter 2 of [4]. Moreover, it is shown in [1] that the least cusp width is the gcd of all the cusp widths, and that the level $m$ is a cusp width and is the lcm of all the cusp widths. Furthermore, the set of cusp widths is closed under gcd and lcm.

\section{LARCHER CONGRUENCE GROUPS}

In [1], H. Larcher introduced a large class of congruence subgroups of the modular groups. These groups generalize the classical congruence groups $\Gamma(n), \Gamma_{0}(n)$ and $\Gamma_{1}(n)$. To describe them we follow the treatment of [1].

Let $m$ be a positive integer and $d$ a positive divisor of $m$. Write $m / d=h^{2} n$, with $n$ square-free. Let $\varepsilon$ and $\chi$ be positive integers such that $\varepsilon \mid h$ and $\chi \mid \operatorname{gcd}\left(d \varepsilon, m / d \varepsilon^{2}\right)$, and let $\tau \in\{1,2, \cdots, \chi\}$. Define the following:

$$
\Gamma_{\tau}(m ; m / d, \varepsilon, \chi)=\left\{ \pm\left(\begin{array}{cc}
1+\frac{m}{\varepsilon \chi} \alpha & d \beta \\
\frac{m}{\chi} \gamma & 1+\frac{m}{\varepsilon \chi} \delta
\end{array}\right), \quad \gamma \equiv \tau \alpha \quad \bmod \chi\right\} / \pm 1,
$$

where $\alpha, \beta, \gamma$ and $\delta$ are integers, and the matrices involved have determinant 1. Then, $\Gamma_{\tau}(m ; m / d, \varepsilon, \chi)$ is a congruence group of level $m$, except for $\Gamma_{1}(4 ; 2,1,2)$ and $\Gamma_{1}(8 ; 8,2,2)$, which are respectively $\Gamma(2)$ of level 2 and $\Gamma_{0}(4)$ of level 4 . Moreover, $d$ is the least cusp width and corresponds to the cusp at $\infty$, while $m$ is 
the width of the cusp 0 . In particular if $m$ is square-free, then $\varepsilon=\chi=1$ and $\Gamma_{\tau}(m ; m / d, \varepsilon, \chi)=\Gamma_{1}(m) \cap \Gamma(d)$. The cusp widths can be determined in terms of the rational presentation of the cusps and the various data attached to these groups. More interestingly, the Larcher groups describe the cusp widths of any congruence group in the following way: Let $\Gamma$ be a congruence group of level $m$, and let $d$ be the least cusp width in $\Gamma$. It is possible to conjugate $\Gamma$ by a matrix in the modular group such that the width of $\infty$ becomes $d$ and the width of 0 becomes $m$. Note that a modular conjugacy only permutes the cusp widths, however, a nonmodular conjugacy often changes the set of cusp widths. According to Section 3 in [1, for suitable $\varepsilon, \chi$ and $\tau$, the Larcher group $\Gamma_{\tau}(m ; m / d, \varepsilon, \chi)$ is a congruence group having the properties:

1. $\Gamma_{\tau}(m ; m / d, \varepsilon, \chi)$ is a subgroup of $\Gamma$.

2. The widths of any cusp (rational or $\infty$ ) are the same with respect to $\Gamma$ and to $\Gamma_{\tau}(m ; m / d, \varepsilon, \chi)$.

We refer to $\Gamma_{\tau}(m ; m / d, \varepsilon, \chi)$ as the Larcher group corresponding to $\Gamma$.

The cusp widths in a Larcher congruence group $\Gamma_{\tau}(m ; m / d, \varepsilon, \chi)$ are given in the following way according to Lemma 7 of [1]: The cusp width of $\infty$ is $d$, and if $a / b$ is a rational number with $\operatorname{gcd}(a, b)=1$, then its width is given by

$$
\frac{d \sigma}{\operatorname{gcd}(\sigma, \varepsilon \chi, b-\tau a \varepsilon)}, \quad \text { with } \quad \sigma=\frac{m}{\operatorname{gcd}(d b, m)},
$$

except for $\Gamma_{1}(4 ; 2,1,2)=\Gamma(2)$ and $\Gamma_{1}(8 ; 8,2,2)$, which are equal to $\Gamma(2)$ and $\Gamma_{0}(4)$ respectively.

\section{TORSION-FREE GENUS 0 GROUPS}

Let $\Gamma$ be any subgroup of $\mathrm{PSL}_{2}(\mathbb{Z})$ of finite index $\mu$ and genus $g$. Let $\nu_{k}(k=2,3)$ be the number of inequivalent elliptic fixed points of order $k$ (which are fixed by transformations of order $k$ ), and let $h$ be the number of the inequivalent cusps, which we also call the parabolic class number. Then the Riemann-Hurwitz formula yields

$$
g=1+\frac{\mu}{12}-\frac{\nu_{2}}{4}-\frac{\nu_{3}}{3}-\frac{h}{2} .
$$

Suppose that $\Gamma$ has $r$ conjugacy classes of elliptic cyclic subgroups of orders $m_{1}, \ldots, m_{r}\left(m_{i} \in\{2,3\}\right)$. Then we say that $\Gamma$ has signature $\left(g ; m_{1}, \ldots, m_{r} ; h\right)$. The structure of the group can be determined by its signature. In fact the group $\Gamma$ has a presentation:

Generators:

$$
A_{1}, B_{1}, \ldots, A_{g}, B_{g}, E_{1}, \ldots, E_{r}, P_{1}, \ldots, P_{h} \text {. }
$$

Relations:

$$
E_{1}^{m_{1}}=\ldots=E_{r}^{m_{r}}=P_{1} \ldots P_{h} E_{1} \ldots E_{r} A_{1} B_{1} A_{1}^{-1} B_{1}^{-1} \ldots A_{g} B_{g} A_{g}^{-1} B_{g}^{-1}=1 .
$$

The generators $P_{i}$ are parabolic, the $E_{i}$ are elliptic and $A_{i}$ and $B_{i}$ are hyperbolic. We note that what has been said so far in this section generalizes to any finitely generated Fuchsian group. 
From the presentation of the group $\Gamma$, it is clear that if $\Gamma$ is torsion-free (that is to say, it has no elliptic elements), and if it is of genus zero, then it can be generated by parabolic elements only. In such a case, it follows from (4.1) that

$$
\mu=6(h-2) \text {. }
$$

The modular group $\mathrm{PSL}_{2}(\mathbb{Z})$ is the free product of a cyclic group of order 2 and a cyclic group of order 3. Using Kurosh's theorem, a torsion-free subgroup of finite index $\Gamma$ is a free group. When $\Gamma$ has genus zero, its rank is $h-1$, where $h$ is the parabolic class number of $\Gamma$. For a positive integer $m$, it is not difficult to see that $\Gamma(m)$ is torsion-free for $m \geq 2, \Gamma_{1}(m)$ is torsion-free for $m>2$ and a trace argument shows that $\Gamma_{0}(m)$ is torsion-free if and only if -1 and -3 are not squares modulo $m$.

Proposition 4.1. Every torsion-free genus zero congruence subgroup of $P S L_{2}(\mathbb{Z})$ is conjugate by an element of $P S L_{2}(\mathbb{Z})$ to a Larcher congruence group.

Proof. Let $\Gamma$ be a torsion-free genus 0 congruence subgroup of level $m$. Up to a modular group conjugacy, we can assume that the least cusp width $d$ corresponds to $\infty$, and that $m$ corresponds to the cusp 0 . Let $\Gamma_{\tau}(m ; m / d, \varepsilon, \chi)$ be the corresponding Larcher group. According to the previous section, the stabilizers of each cusp with respect to both groups are the same. It follows that every parabolic generator of $\Gamma$, which is a generator of the stabilizer of the corresponding cusp, is also in $\Gamma_{\tau}(m ; m / d, \varepsilon, \chi)$. Since $\Gamma$ is torsion-free and genus 0 , it can be generated by parabolic elements only. Therefore $\Gamma=\Gamma_{\tau}(m ; m / d, \varepsilon, \chi)$.

In the next section, we will list the congruence groups which are torsion-free and genus zero. It will be easy to decide whether they are genus 0 simply because they are all conjugate to one of the groups of the form $\Gamma(n), \Gamma_{0}(n)$ or $\Gamma_{1}(n)$ which are mentioned in the introduction, or are $\Gamma_{0}(16) \cap \Gamma_{1}(8)$ and $\Gamma_{1}(8) \cap \Gamma(2)$. We therefore need the following result:

Proposition 4.2. The groups $\Gamma_{0}(16) \cap \Gamma_{1}(8)$ and $\Gamma_{1}(8) \cap \Gamma(2)$ are of genus zero.

Proof. The two groups are conjugate by $\tau \rightarrow 2 \tau$; we will deal only with $\Gamma_{0}(16) \cap$ $\Gamma_{1}(8)$. It is not difficult to see that this group has index 48 in $\operatorname{PSL}_{2}(\mathbb{Z})$. As a subgroup of $\Gamma_{0}(16)$, it is torsion-free. Thus, using the formula (4.1) to prove that the genus is 0 , we only need to show that the parabolic class number $h$ is 10 . The group $\Gamma_{0}(16) \cap \Gamma_{1}(8)$ coincides with the Larcher group $\Gamma_{1}(16 ; 16,1,2)$, for which we can establish the widths of all cusps using $(\underline{3.2})$. For the cusps 1 and $1 / 3$, the width is 16 , for the cusps $1 / 2$ and $1 / 6$ the width is 4 , for the cusps $1 / 4$ and $1 / 12$ the width is 2 and for the cusps $1 / 8,3 / 8,3 / 16$ and $1 / 16$, the width is $1(1 / 16$ is equivalent to $\infty$ ). We will show that those having the same width are inequivalent, and then we have the 10 inequivalent cusps we are looking for. The cusps 1 and $1 / 3$ are inequivalent modulo $\Gamma_{1}(8)$, otherwise we will have, for some integers $a, b$, $c$ and $d$ satisfying the determinant condition $a+d+8 a d=b c$,

$$
\frac{1+8 a+b}{8 c+1+8 d}=\frac{1}{3}
$$

The numerator and denominator are relatively prime since the product

$$
\left(\begin{array}{cc}
1+8 a & b \\
8 c & 1+8 d
\end{array}\right)\left(\begin{array}{ll}
1 & 0 \\
1 & 1
\end{array}\right)=\left(\begin{array}{cc}
1+8 a+b & b \\
8 c+1+8 d & 1+8 d
\end{array}\right)
$$


has determinant 1 ; this contradicts (4.5). If the cusps $1 / 2$ and $1 / 6$ are equivalent modulo $\Gamma_{0}(16) \cap \Gamma_{1}(8)$, then 1 and $1 / 3$ will be equivalent modulo $\Gamma_{1}(8) \cap \Gamma(2)$, but we have just seen that they are not equivalent modulo $\Gamma_{1}(8)$. If the cusps $1 / 4$ and $1 / 12$ are equivalent modulo $\Gamma_{0}(16) \cap \Gamma_{1}(8)$, then for some $a, b, c$ and $d$ we have

$$
\frac{1+8 a+4 b}{16 c+4+32 d}=\frac{1}{12} \text {. }
$$

This is equivalent to $3(1+8 a+4 d)=1+4 c+8 d$, which is impossible mod 4. Similarly, the cusps $1 / 8$ and $3 / 8$ are inequivalent modulo $\Gamma_{1}(8)$ and so they are modulo $\Gamma_{0}(16) \cap \Gamma_{1}(8)$. The cusps $3 / 16$ and $1 / 16$ are inequivalent modulo $\Gamma_{0}(16) \cap \Gamma_{1}(8)$ because $3 / 8$ and $1 / 8$ are inequivalent modulo $\Gamma_{1}(8)$. Finally, none of the cusps $1 / 8$ and $3 / 8$ is equivalent to one of the cusps $1 / 16$ and $3 / 16$ under $\Gamma_{0}(16)$.

\section{The COMPlete List}

Theorem 5.1. Up to modular conjugacy, there are exactly 33 congruence subgroups of the modular group which are torsion-free and genus zero, all of which are given in Table 1.

\section{THE PROOF}

According to Proposition 4.1, it is enough to determine all the Larcher congruence groups which are torsion-free and genus 0 . We start with the following

Proposition 6.1. If $\Gamma_{\tau}(m ; m / d, \varepsilon, \chi)$ is of genus zero, then

$$
d \varepsilon \leq 5, \quad \frac{m d}{\chi} \leq 25, \quad \frac{m}{\varepsilon \chi} \leq 12 .
$$

Proof. It is clear that $\Gamma_{\tau}(m ; m / d, \varepsilon, \chi) \subseteq \Gamma_{0}(m / \chi) \cap \Gamma_{1}(m / \varepsilon \chi) \cap \Gamma(d)$ since $d$ divides $m / \varepsilon \chi$. Also $\Gamma_{0}(m / \chi) \cap \Gamma(d)$ is conjugate by $\left(\begin{array}{ll}d & 0 \\ 0 & 1\end{array}\right)$ to $\Gamma_{0}(m d / \chi) \cap \Gamma_{1}(d)$ and $\Gamma_{0}(m / \chi) \cap$ $\Gamma_{1}(m / \varepsilon \chi) \cap \Gamma(d)$ is conjugate by $\left(\begin{array}{cc}1 & 0 \\ 0 & \varepsilon\end{array}\right)$ to $\Gamma_{1}(m / \varepsilon \chi) \cap \Gamma(d \varepsilon)$ since $d \varepsilon$ divides $m / \varepsilon \chi$. A necessary condition to have $\Gamma_{\tau}(m ; m / d, \varepsilon, \chi)$ of genus zero is that the groups $\Gamma_{0}(m d / \chi), \Gamma_{1}(m / \varepsilon \chi)$ and $\Gamma(d \varepsilon)$ are all of genus zero. This yields (6.1).

We see that $d \leq 5$. The Proof of Theorem 5.1 will be detailed in five lemmas according to the value of $d$.

Lemma 6.2. There is only one torsion-free genus 0 congruence group with $d=5$. Namely $\Gamma(5)$ of index 60.

Proof. If $d=5$, from (6.1) we get $\varepsilon=1$. Since $\chi \mid d \varepsilon$, we have $\chi=1$ or 5 . If $\chi=5$, then since $d \chi \mid m$ and $m d / \chi \leq 25$, we have $m=25$. We end up with the groups $\Gamma_{\tau}(25 ; 5,1,5)$ for $\tau \in\{1,2,3,4,5\}$ which consist of the transformations

$$
A=\left(\begin{array}{cc}
1+5 \alpha & 5 \beta \\
5 \gamma & 1+5 \delta
\end{array}\right), \quad \operatorname{det}(A)=1 \text { and } \gamma \equiv \tau \alpha \quad \bmod 5 .
$$

Conjugating by $\left(\begin{array}{cc}1 & -\tau \\ 0 & 1\end{array}\right)$ if $\tau=1,4$ or by $\left(\begin{array}{ll}1 & \tau \\ 0 & 1\end{array}\right)$ if $\tau=2,3$, and then by $\left(\begin{array}{ll}1 & 0 \\ 0 & 5\end{array}\right)$ we obtain $\Gamma_{1}(25)$ which is not of genus 0 . While if $\tau=5$, then the group is $\Gamma_{0}(25) \cap \Gamma(5)$ which is conjugate to $\Gamma_{0}(125) \cap \Gamma_{1}(5)$ by $\left(\begin{array}{ll}1 & 0 \\ 0 & 5\end{array}\right)$ which is not of genus zero. If $\chi=1$, then from $m d / \chi \leq 25$ we get $m=5$ and $\tau=1$ and so the group is simply $\Gamma(5)$ which is genus zero and has index 60 in the modular group. 


\section{TABle 1.}

\begin{tabular}{|c|c|c|}
\hline Index & Level & Group \\
\hline 6 & $\begin{array}{l}2 \\
4\end{array}$ & $\begin{array}{l}\Gamma(2) \\
\Gamma_{0}(4)\end{array}$ \\
\hline 12 & $\begin{array}{l}3 \\
4 \\
5 \\
6 \\
8 \\
9\end{array}$ & $\begin{array}{l}\Gamma(3) \\
\Gamma_{0}(4) \cap \Gamma(2) \\
\Gamma_{1}(5) \\
\Gamma_{0}(6) \\
\Gamma_{0}(8) \\
\Gamma_{0}(9)\end{array}$ \\
\hline 24 & $\begin{array}{l}4 \\
6 \\
7\end{array}$ & $\begin{array}{l}\Gamma(4) \\
\Gamma_{0}(3) \cap \Gamma(2) \\
\Gamma_{1}(7) \\
\Gamma_{1}(8), \Gamma_{0}(8) \cap \Gamma(2),\left\{ \pm\left(\begin{array}{cc}1+4 a & 2 b \\
4 c & 1+4 d\end{array}\right), a \equiv c \quad \bmod 2\right\} \\
\Gamma_{0}(12) \\
\Gamma_{0}(16),\left\{ \pm\left(\begin{array}{cc}1+4 a & b \\
8 c & 1+4 d\end{array}\right), \quad a \equiv c \quad \bmod 2\right\}\end{array}$ \\
\hline 36 & $\begin{array}{l}10 \\
18\end{array}$ & $\begin{array}{l}\Gamma_{0}(2) \cap \Gamma(3) \\
\Gamma_{1}(9),\left\{ \pm\left(\begin{array}{cc}1+3 a & 3 b \\
3 c & 1+3 d\end{array}\right), \quad a \equiv c \quad \bmod 3\right\} \\
\Gamma_{1}(10) \\
\Gamma_{0}(18) \\
\left\{ \pm\left(\begin{array}{cc}1+3 a & b \\
9 c & 1+3 d\end{array}\right), \quad a \equiv c \quad \bmod 3\right\}\end{array}$ \\
\hline 48 & 12 & $\begin{array}{l}\Gamma_{1}(8) \cap \Gamma(2),\left\{ \pm\left(\begin{array}{cc}1+4 a & 4 b \\
4 c & 1+4 d\end{array}\right), \quad a \equiv c \quad \bmod 2\right\} \\
\Gamma_{1}(12),\left\{ \pm\left(\begin{array}{cc}1+6 a & 2 b \\
6 c & 1+6 d\end{array}\right), \quad a \equiv c \quad \bmod 2\right\} \\
\Gamma_{0}(16) \cap \Gamma_{1}(8),\left\{ \pm\left(\begin{array}{cc}1+4 a & 2 b \\
8 c & 1+4 d\end{array}\right), \quad a \equiv c \bmod 2\right\} \\
\left\{ \pm\left(\begin{array}{cc}1+6 a & b \\
12 c & 1+6 d\end{array}\right), \quad a \equiv c \quad \bmod 2\right\} \\
\left\{ \pm\left(\begin{array}{cc}1+4 a & b \\
16 c & 1+4 d\end{array}\right), \quad a \equiv c \quad \bmod 2\right\}\end{array}$ \\
\hline 60 & $\begin{array}{r}5 \\
25\end{array}$ & $\begin{array}{l}\Gamma(5) \\
\Gamma_{0}(25) \cap \Gamma_{1}(5)\end{array}$ \\
\hline
\end{tabular}


Lemma 6.3. If $d=4$, there are exactly 2 Larcher groups of genus 0 . Namely $\Gamma(4)$ of index 24 and the group $\Gamma_{1}(8 ; 2,1,2)$ of transformations $\left(\begin{array}{cc}1+4 a & 4 d \\ 4 c & 1+4 d\end{array}\right)$ with $a \equiv c$ mod 2 of index 48.

Proof. From $d \varepsilon \leq 5$ we have $\varepsilon=1$, and $\chi \mid 4$ so that $\chi=1,2$ or 4 . If $\chi=1$, then $d \mid m$ and $m d / \chi \leq 25$ yields $m=4$, in which case the group is clearly $\Gamma(4)$ which has index 24 in $\mathrm{PSL}_{2}(\mathbb{Z})$. If $\chi=2$, then from $m d / \chi \leq 25$ and $d \chi \mid m$ we have $m=8$. The possible values for $\tau$ are 1 and 2 . In the first case we have $\Gamma_{1}(8 ; 2,1,2)$ which is conjugate by $\left(\begin{array}{ll}1 & 1 \\ 0 & 2\end{array}\right)$ to $\Gamma_{1}(8) \cap \Gamma(2)$ which is genus zero by Proposition 4.2 If $\tau=2$, then we have $\Gamma_{2}(8 ; 2,1,2)$ which is simply $\Gamma_{0}(8) \cap \Gamma(4)$ which is conjugate by $\left(\begin{array}{ll}1 & 0 \\ 0 & 4\end{array}\right)$ to $\Gamma_{0}(32)$ and therefore not of genus zero. It remains to look at the case $\chi=4$ which, together with $m d / \chi \leq 25$ and $d \chi \mid m$, gives $m=16$. For $\tau=1,2,3,4$ one can easily see that the corresponding Larcher groups are respectively conjugate to $\Gamma_{1}(16), \Gamma_{0}(32), \Gamma_{1}(16)$ and $\Gamma_{0}(64)$, none of which is of genus zero.

Lemma 6.4. If $d=3$, there are exactly three Larcher groups of genus zero. Namely $\Gamma(3)$ of index $12, \Gamma_{0}(2) \cap \Gamma(3)$ of index 36 and the group $\Gamma_{1}(9 ; 3,1,3)$ of transformations $\left(\begin{array}{ccc}1+3 a & 3 b \\ 3 c & 3 d\end{array}\right)$ with $a \equiv c \bmod 3$ of index 36 .

Proof. From $d \varepsilon \leq 5$, we still have $\varepsilon=1$, and then $\chi=1$ or 3 . If $\chi=1, m d / \chi \leq 25$ yields $m \leq 8$, and $d \mid m$ yields $m=3$ or 6 . In the first case, the group is simply $\Gamma(3)$, in the second case, it is $\Gamma_{0}(6) \cap \Gamma(3)=\Gamma_{0}(2) \cap \Gamma(3)$ which is of genus zero since it is conjugate to $\Gamma_{0}(18)$. If $\chi=3$, the possible values of $m$ are 9 and 18 . If $m=9$, then for $\tau=1,2$ we have the group $\Gamma_{\tau}(9 ; 3,1,3)$ of transformations $\left(\begin{array}{cc}1+3 a & 3 b \\ 3 c & 3 d\end{array}\right)$ with the condition $a \equiv c \bmod 3$ if $\tau=1$ and $a \equiv-c \bmod 3$ if $\tau=2$. But these two groups are clearly conjugate by $\left(\begin{array}{ll}1 & 1 \\ 0 & 1\end{array}\right)$ which is in the modular group; we keep only the case $\tau=1$. The corresponding group is of genus zero since it is conjugate to $\Gamma_{1}(9)$ by $\left(\begin{array}{cc}1 & -1 \\ 0 & 3\end{array}\right)$. If $\tau=3$, then we are dealing with the group $\Gamma_{3}(9 ; 3,1,3)=\Gamma_{0}(9) \cap \Gamma(3)$ which is conjugate to $\Gamma_{0}(27)$, and therefore not of genus zero. It remains to look at the case $m=18$. If $\tau=1$ or 2 , then the corresponding Larcher group is conjugate to $\Gamma_{1}(18)$, and if $\tau=3$ it is conjugate to $\Gamma_{0}(54)$ and none of these is genus zero.

Lemma 6.5. If $d=2$, there are exactly 8 Larcher groups of genus zero. Namely $\Gamma(2)$ of index $6, \Gamma_{0}(4) \cap \Gamma(2)$ of index $12, \Gamma_{0}(3) \cap \Gamma(2), \Gamma_{0}(8) \cap \Gamma(2)$ and $\Gamma_{1}(8 ; 4,1,2)$ of index 24 and $\Gamma_{1}(8) \cap \Gamma(2), \Gamma_{1}(12 ; 6,1,2)$ and $\Gamma_{1}(16 ; 8,2,2)$ of index 48.

Proof. If $d=2$, then from $d \varepsilon \leq 5$ we get $\varepsilon=1$ or 2 .

(i) $\varepsilon=1: \chi$ divides $d=2$ which divides $m$.

If $\chi=1$, then from $m d / \chi \leq 25$ we get $m \leq 12$ and $m$ is even. In all cases $\tau$ is 1 , and we can read off the following Larcher groups for each even value of $m$ from 2 to 12: $\Gamma(2), \Gamma_{0}(4) \cap \Gamma(2), \Gamma_{0}(6) \cap \Gamma(2)=\Gamma_{0}(3) \cap \Gamma(2), \Gamma_{1}(8) \cap \Gamma(2), \Gamma_{1}(10) \cap \Gamma(2)$ and $\Gamma_{1}(12) \cap \Gamma(2)$. The last two are not genus 0 because they are respectively conjugate to $\Gamma_{0}(20) \cap \Gamma_{1}(10)$ and $\Gamma_{0}(24) \cap \Gamma_{1}(12)$. The groups $\Gamma_{0}(4) \cap \Gamma(2)$ and $\Gamma_{0}(3) \cap \Gamma(2)$ are both genus zero because they are respectively conjugate by $\left(\begin{array}{ll}2 & 0 \\ 0 & 1\end{array}\right)$ to $\Gamma_{0}(8)$ and $\Gamma_{0}(12)$.

If $\chi=2$, then $m$ is multiple of 4 less than 25. If $m=4$ and $\tau=1$, then we are dealing with the first exceptional Larcher group (in the sense that the level is not immediately apparent); this group is $\Gamma_{1}(4 ; 2,1,2)=\Gamma(2)$. While if $\tau=2$, then the group is $\Gamma_{2}(4 ; 2,1,2)=\Gamma_{0}(4) \cap \Gamma(2) 11$ If $m=8$, then $\chi=1$ gives $\Gamma_{1}(8 ; 4,1,2)$ which

\footnotetext{
${ }^{1}$ The reason we get this group twice is that $\Gamma_{0}(4)=\Gamma_{1}(4)$. The same phenomenon will occur whenever we have to deal with $\Gamma_{0}(4)$ or $\Gamma_{0}(6)=\Gamma_{1}(6)$.
} 
is genus 0 because it is conjugate to $\Gamma_{1}(8)$ by $\left(\begin{array}{ll}1 & 1 \\ 0 & 2\end{array}\right)$, while $\tau=2$ gives $\Gamma_{0}(8) \cap \Gamma(2)$, a conjugate of $\Gamma_{0}(16)$ and hence of genus 0 . If $m=12$, then only $\tau=1$ gives a genus 0 group which is $\Gamma_{1}(12 ; 6,1,2)$, a conjugate to $\Gamma_{1}(12)$. For $m=16,20,24$, it is easy to see that the groups produced are not genus zero.

(ii) $\varepsilon=2: \chi$ divides $d \varepsilon=4$ so that $\chi=1,2,4$. Since $\chi d \varepsilon^{2} \mid m$ and $m d / \chi \leq 25$, the only possibilities are $(\chi=1, m=8),(\chi=2, m=16)$ or $(\chi=4, m=32)$. The first case corresponds to $\Gamma_{0}(8) \cap \Gamma_{1}(4) \cap \Gamma(2)=\Gamma_{0}(8) \cap \Gamma(2)$. For the second case, if $\tau=1$, then we have $\Gamma_{1}(16 ; 8,1,2)$ which is of genus 0 because it is conjugate to $\Gamma_{1}(8) \cap \Gamma(2)$ by $\left(\begin{array}{cc}1 & 1 / 2 \\ 0 & 1\end{array}\right)$, and $\tau=2$ gives $\Gamma_{0}(16) \cap \Gamma(2)$ which is not of genus 0 since it is conjugate to $\Gamma_{0}(32)$. For the third case, all four values of $\tau$ give nonzero genus.

Notice that in the previous lemmas, the genus zero groups are all torsion-free since they are contained in $\Gamma(m)$ for $m=2,3,4$ or 5 . However, this is no longer the case with $d=1$.

Lemma 6.6. If $d=1$, there are exactly 19 Larcher groups which are torsionfree and genus zero. Namely, $\Gamma_{0}(m)$ for $m=4,6,8,9,12,16,18, \Gamma_{1}(m)$ for $m=$ $5,7,8,9,10,12, \Gamma_{0}(16) \cap \Gamma_{1}(8), \quad \Gamma_{0}(25) \cap \Gamma_{1}(5), \quad \Gamma_{1}(16 ; 16,2,2), \Gamma_{1}(24 ; 24,2,2)$, $\Gamma_{1}(27 ; 27,3,3)$ and $\Gamma_{1}(32,32,4,2)$.

Proof. (i) $\varepsilon=1$ : We have $\chi=1$, and $m / \chi \varepsilon \leq 12$ yields $m \leq 12$, and the groups are all of the form $\Gamma_{1}(m)$. We have to exclude 11 because of the genus 0 property, and 1, 2 and 3 because of the torsion-free property.

(ii) $\varepsilon=2$ : If $\chi=1$, then $m \leq 24$ and $4 \mid m$. The groups are of the form $\Gamma_{0}(m) \cap \Gamma_{1}(m / 2)$. This produces $\Gamma_{0}(4), \Gamma_{0}(8), \Gamma_{0}(12), \Gamma_{0}(16) \cap \Gamma_{1}(8)$ which are all of genus zero and torsion-free, however, $m=20$ or 24 does not give a genus zero group. If $\chi=2$, then $m \leq 48$ and $m$ must be divisible by $\varepsilon^{2} \chi=8$. It is not difficult to exclude $m=32,40$ and 48 . For $m=24, \tau=1$ gives $\Gamma_{1}(24 ; 24,2,2)$ which is conjugate to $\Gamma_{1}(12)$ by $\left(\begin{array}{cc}1 & 1 / 2 \\ 0 & 1\end{array}\right)$, and so it is of genus 0 , and $\tau=2$ gives $\Gamma_{0}(24) \cap \Gamma_{1}(12)$ which is not of genus 0 . If $m=16$, then $\tau=2$ gives $\Gamma_{0}(16)$ and $\tau=1$ yields $\Gamma_{1}(16 ; 16,2,2)$ which is conjugate to $\Gamma_{1}(8)$. If $m=8$, then $\tau=2$ yields $\Gamma_{0}(8)$ and $\tau=1$ corresponds to the second exceptional Larcher group, namely $\Gamma_{1}(8 ; 8,2,2)$ which is just $\Gamma_{0}(4)$.

(iii) $\varepsilon=3: \chi=1$ or 3 , and $\varepsilon^{2} \chi \mid m$ gives $9 \chi \mid m$. If $\chi=1$, then $m d / \chi \leq 25$ yields $m \leq 25$, so that $m=9,18$; these correspond to the groups $\Gamma_{0}(9) \cap \Gamma_{1}(3)=\Gamma_{0}(9)$ and $\Gamma_{0}(18) \cap \Gamma_{1}(6)=\Gamma_{0}(18)$. If $\chi=3$, then $m \leq 75$ and $27 \mid m$, so that $m=27$ or 54. For $m=54$, the groups corresponding to $\tau=1$ or 2 are conjugate by $\left(\begin{array}{cc}1 & \tau / 3 \\ 0 & 1\end{array}\right)$ to $\Gamma_{1}(18)$ and $\tau=3$ gives $\Gamma_{0}(54)$ and none of these is genus zero. For $m=27$, $\tau=3$ yields $\Gamma_{0}(27)$ which is not of genus 0 . However, $\tau=1$ or 2 yield two genus zero groups which are conjugate to $\Gamma_{1}(9)$ by $\left(\begin{array}{cc}1 & -\tau / 3 \\ 0 & 1\end{array}\right)$. Note that $\Gamma_{1}(27 ; 27,3,3)$ is conjugate to $\Gamma_{2}(27 ; 27,3,3)$ by $\left(\begin{array}{ll}2 & -1 \\ 3 & -1\end{array}\right)$ which is in the modular group, and they are also conjugate by $\left(\begin{array}{cc}1 & 1 / 3 \\ 0 & 1\end{array}\right)$.

(iv) $\varepsilon=4:$ In this case $\chi=1,2,4$ and the conditions $\chi \varepsilon^{2} \mid m$ and $m / \chi \leq 25$ give the following: If $\chi=1$, then $m=16$ and the group is $\Gamma_{0}(16)$. If $\chi=2$, then $m \leq 50$ and $32 \mid m$, which yields $m=32$. Now, for $\tau=1$ we have the group $\Gamma_{1}(32 ; 32,4,2)$ which is conjugate to $\Gamma_{0}(16) \cap \Gamma_{1}(8)$ by $\left(\begin{array}{cc}1 & -1 / 4 \\ 0 & 1\end{array}\right)$ and hence of genus 0 , and $\tau=2$ gives the group $\Gamma_{0}(32)$ which is not of genus zero. If $\chi=4$, then $m \leq 100$ and $64 \mid \mathrm{m}$ so that $m=64$. In this case $\tau=1,3$ give groups that are conjugate to $\Gamma_{1}(16)$, $\tau=2,4$ give groups that are subgroups of $\Gamma_{0}(32)$, and none of these is of genus 0 . 
(v) $\varepsilon=5:$ In this case $\chi=1$ or 5 . We have $25 \chi=\chi \varepsilon^{2} \mid m$ and $m / \chi \leq 25$, yielding $m=25 \chi$. If $\chi=1$, then $m=25$ and the group is $\Gamma_{0}(25) \cap \Gamma_{1}(5)$ which is conjugate to $\Gamma(5)$ and hence of genus 0 . If $\chi=5$, then $m=125$, and $\tau=1,2,3$ or 4 give a group that is conjugate to $\Gamma_{1}(25)$ by $\left(\begin{array}{cc}1 & -\tau / 5 \\ 0 & 1\end{array}\right)$, and $\tau=5$ gives $\Gamma_{0}(125) \cap \Gamma_{1}(5)$, and none of these groups is of genus 0 .

This concludes the proof of the classification.

\section{Cusp Widths}

TABLE 2 .

\begin{tabular}{|r|l|l|}
\hline index & Group & Cusp widths \\
\hline 6 & $\Gamma(2)$ & $2-2-2$ \\
& $\Gamma_{0}(4)$ & $4-1-1$ \\
\hline 12 & $\Gamma(3)$ & $3-3-3-3$ \\
& $\Gamma_{0}(4) \cap \Gamma(2)$ & $4-4-2-2$ \\
& $\Gamma_{1}(5)$ & $5-5-1-1$ \\
& $\Gamma_{0}(6)$ & $6-3-2-1$ \\
& $\Gamma_{0}(8)$ & $8-2-1-1$ \\
& $\Gamma_{0}(9)$ & $9-1-1-1$ \\
\hline 24 & $\Gamma(4)$ & $4-4-4-4-4-4$ \\
& $\Gamma_{0}(3) \cap \Gamma(2)$ & $6-6-6-2-2-2$ \\
& $\Gamma_{1}(7)$ & $7-7-7-1-1-1$ \\
& $\Gamma_{1}(8)$ & $8-8-4-2-1-1$ \\
& $\Gamma_{0}(8) \cap \Gamma(2)$ & $8-8-2-2-2-2$ \\
& $\Gamma_{1}(8 ; 4,1,2)$ & $8-4-4-4-2-2$ \\
& $\Gamma_{0}(12)$ & $12-4-3-3-1-1$ \\
& $\Gamma_{0}(16)$ & $16-4-1-1-1-1$ \\
& $\Gamma_{1}(16 ; 16,2,2)$ & $16-2-2-2-1-1$ \\
\hline 36 & $\Gamma_{0}(2) \cap \Gamma(3)$ & $6-6-6-6-3-3-3-3$ \\
& $\Gamma_{1}(9)$ & $9-9-9-3-3-1-1-1$ \\
& $\Gamma_{1}(9 ; 3,1,3)$ & $9-9-3-3-3-3-3-3$ \\
& $\Gamma_{1}(10)$ & $10-10-5-5-2-2-1-1$ \\
& $\Gamma_{0}(18)$ & $18-9-2-2-2-1-1-1$ \\
& $\Gamma_{1}(27 ; 27,3,3)$ & $27-3-1-1-1-1-1-1$ \\
\hline 48 & $\Gamma_{1}(8) \cap \Gamma(2)$ & $8-8-8-8-4-4-2-2-2-2$ \\
& $\Gamma_{1}(8 ; 2,1,2)$ & $8-8-4-4-4-4-4-4-4-4$ \\
& $\Gamma_{1}(12)$ & $12-12-6-4-4-3-3-2-1-1$ \\
& $\Gamma_{1}(12 ; 6,1,2)$ & $12-6-6-6-6-4-2-2-2-2$ \\
& $\Gamma_{0}(16) \cap \Gamma_{1}(8)$ & $16-16-4-4-2-2-1-1-1-1$ \\
& $\Gamma_{1}(16 ; 8,2,2)$ & $16-16-2-2-2-2-2-2-2-2$ \\
& $\Gamma_{1}(24 ; 24,2,2)$ & $24-8-3-3-3-3-1-1-1-1$ \\
& $\Gamma_{1}(32 ; 32,4,2)$ & $32-8-1-1-1-1-1-1-1-1$ \\
\hline 60 & $\Gamma(5)$ & $5-5-5-5-5-5-5-5-5-5-5-5$ \\
& $\Gamma_{0}(25) \cap \Gamma_{1}(5)$ & $25-25-1-1-1-1-1-1-1-1-1-1$ \\
\hline
\end{tabular}

In Table 2 we give the list of the cusp widths of all the torsion-free genus zero congruence subgroups of the modular group. We recall that the cusp widths satisfy 
the following properties:

- The width of 0 (which is the level) is the lcm of all widths.

- The width of $\infty$ is the gcd of all widths.

- The sum of all widths equals the index of the group in $\mathrm{PSL}_{2}(\mathbb{Z})$.

These widths were computed using the above properties in some cases, and in other cases by finding a set of inequivalent cusps for the group and using (3.2).

\section{REFERENCES}

[1] Larcher, H. The cusp amplitudes of the congruence subgroups of the classical modular group. II. Illinois J. Math. 28 (1984), 312-338. MR 85i:11034

[2] McKay, J., Sebbar, A. Fuchsian groups, Schwarzians, and theta functions, C. R. Acad. Sci. Paris, t.327, Serie I, pp.343-348, 1998. MR 2000a:11059

[3] McKay, J., Sebbar, Fuchsian groups, automorphic functions and Schwarzians, Math. Ann. 318 (2000), 255-275. CMP 2001:04

[4] Rankin, Robert A. Modular forms and functions. Cambridge University Press, 1977. MR 58:16518

[5] Sebbar A. Conjugacy classes of torsion-free genus zero congruence subgroups of $\mathrm{PSL}_{2}(\mathbb{R})$, To appear in Duke Math. J.

[6] Shimura, G. Introduction to the arithmetic theory of automorphic functions. Princeton University Press, Princeton, 1971. MR 47:3318

Department of Mathematics and Statistics, University of Ottawa, Ottawa, Ontario, CANADA K1N 6N5

E-mail address: sebbar@mathstat.uottawa.ca 